\title{
GOCE: Ein Gravitationsmesslabor im Satelliten
}

Reiner Rummel

Technische Universität München

Institut für Astronomische und Physikalische Geodäsie

Arcisstrasse 21

80290 München

08928923189

rummel@bv.tum.de

GOCE (Gravity and steady-state Ocean Circulation Explorer) ist der erste Satellit des neu definierten Erdbeobachtungsprogramms "Living Planet“ der Europäischen Raumfahrtagentur ESA. Er wurde im März 2009 gestartet. Seine Aufgabe ist die detailgenaue Vermessung des Erdschwerefelds und des Geoids. Erstmals wird dabei das Messprinzip der Gravitationsgradiometrie eingesetzt, d.h. es werden die Gradienten der Gravitationsbeschleunigung entlang der drei orthogonalen Achsen des Instrumentensystems gemessen.

Ionentriebwerk

Xenontank

Nitrogentank

power supply

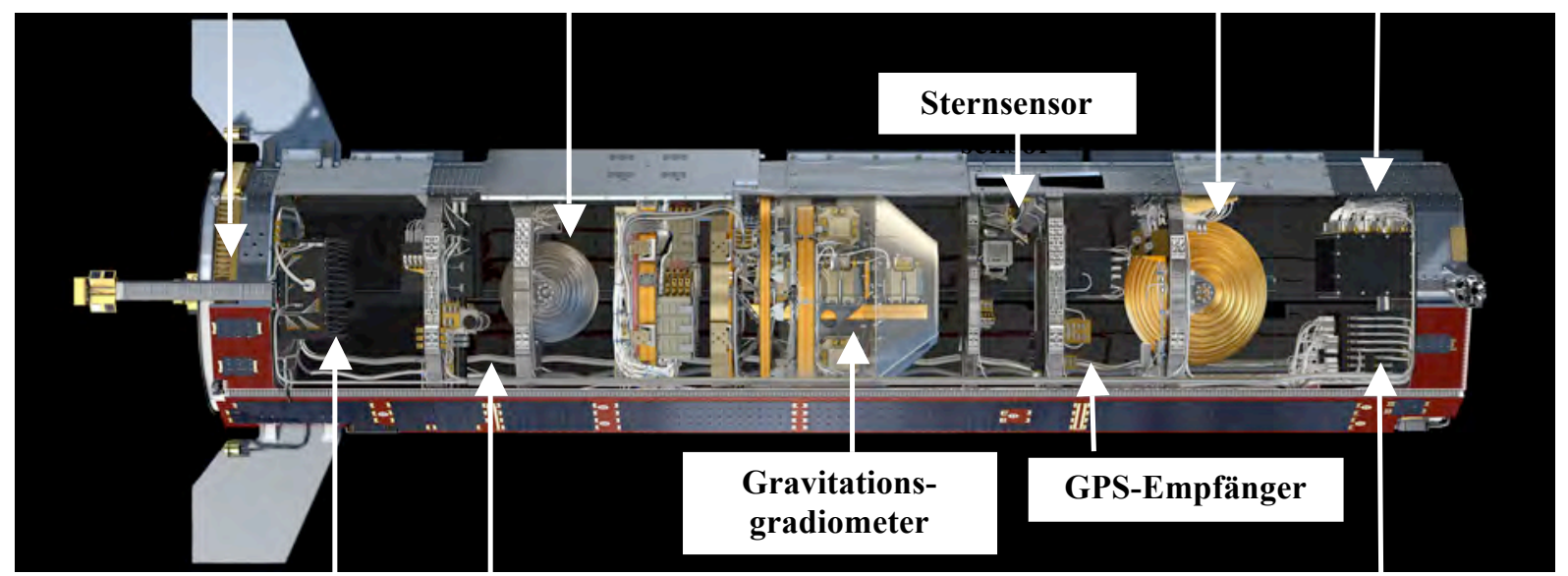

Ionentriebwerk

Magnetfelddrehmomentgeber

Kontrolleinheit

Abbildung 1: Gesamtsensorsystem des Satelliten GOCE (Quelle: ESA). In der Mitte befindet sich das Gravitationsgradiometer

GOCE ist eine erdwissenschaftliche Mission. Die räumlichen Variationen der Erdanziehung liefern wichtige Information über den Gleichgewichtszustand der topographischen Massen und die Dynamik des Erdmantels. Das Geoid, d.h. die Äquipotentialfläche des Erdschwerefelds auf mittlerem Meeresniveau repräsentiert den hypothetischen Gleichgewichtszustand der Weltmeere. Das tatsächliche Niveau der Ozeane und deren zeitliche Veränderungen werden seit ca. 20 Jahren zentimetergenau vom Weltraum aus mit Radaraltimetrie bestimmt. Die Höhendifferenz „tatsächliches Niveau minus Geoid“ ist eine Schlüsselgröße für die Erforschung der Ozeanzirkulationssysteme und des globalen Masse- und Wärmetransports in den Weltmeeren. Von praktischer Bedeutung sind die mit GOCE erreichbare globale Vereinheitlichung aller Höhensysteme und die deutliche Verbesserung der Höheninformation aus GPS.

Gravitationsgradiometrie ist die Messung der Erdanziehung im Zustand der Schwerelosigkeit. Das Gradiometer besteht aus drei orthogonal angeordneten, einachsigen Gradiometern. Jedes von ihnen besteht aus zwei Akzelerometern, jeweils montiert an den Enden einer $50 \mathrm{~cm}$ langen Basis. Der gemeinsame Mittelpunkt des Instruments ist im Massenzentrum des Satelliten. Nur 
im Massenzentrum herrscht perfekte Schwerelosigkeit. Am jeweiligen Ort der sechs Akzelerometer ist die Erdanziehung nicht null aber sehr klein. Sie beträgt ca. ein Millionstel des Wertes von "g" auf der Erde. Die wissenschaftlichen Zielstellungen erfordern eine Messgenauigkeit von ca. 1 Millionstel dieses kleinen Signals. In jedem dieser ultraempfindlichen Beschleunigungssensoren wird ein $320 \mathrm{~g}$ schwerer Quader über ein elektrostatisches Rückkopplungssystem in einem Schwebezustand gehalten. Dem Rückkopplungssignal entspricht die gewünschte Messgröße. Da die Akzelerometer im Labor unter dem Einfluss der Erdanziehung gebaut wurden, konnte man nur jeweils die zwei horizontalen Raumrichtungen ultrapräzise konstruieren, während die vertikale Achse weitaus robuster und daher ungenauer ist.

Das Gravitationsgradiometer ist ein aufwändiges Sensorsystem, siehe Abbildung 1. Nur dadurch lassen sich die hohen Anforderungen an die Genauigkeit des Gradiometers erreichen. Die Akzelerometer des Gradiometers sind fest mit dem Satelliten verbunden. Sie messen daher nicht nur das Gravitationssignal sondern auch die Drehbewegung des Satelliten. Durch eine geeignete Anordnung der empfindlichen Achsen der sechs Beschleunigungsmesser lassen sich aber die Drehbeschleunigungen von den Gravitationsbeschleunigungen trennen. Mit Sternsensoren wird die Orientierung des Instruments bezüglich des Fixsternhimmels ermittelt. Die Kombination von Sternkameramessungen und Drehbeschleunigungen liefert schließlich die auf die Akzelerometer wirkenden Winkelgeschwindigkeiten. Mit einem GPS-Empfänger wird die Bahntrajektorie von GOCE zentimetergenau ermittelt. Zur Verstärkung des Gravitationssignals wurde eine extrem niedrige Bahnhöhe von nur 265km Höhe über der Erde gewählt. Um diese Bahnhöhe zu halten, werden mit den Akzelerometern auch die auf den Satelliten wirkenden Bremskräfte gemessen und über ein lonentriebwerk kompensiert. Die Standregelung des Satelliten erfolgt mit Hilfe von Magnetfeld-Drehmomentgebern. Im Abstand von ca. zwei Monaten wird das Gesamtsystem mit Hilfe eines "Schütteltests“ geeicht. Um Gravitationseffekte des Satelliten selbst auszuschließen, werden enorm hohe Anforderungen an die thermische Stabilität und die Festigkeit der Materialien gestellt.

Das Messsystem arbeitet einwandfrei. Seit November 2009 liefert GOCE hervorragende Schwerefelddaten. Bisher konnten die in den ersten 12 Monaten entstandenen Messreihen vollständig ausgewertet und Modelle des Geoids, siehe Abbildung 2, und des Erdschwerefelds berechnet werden.

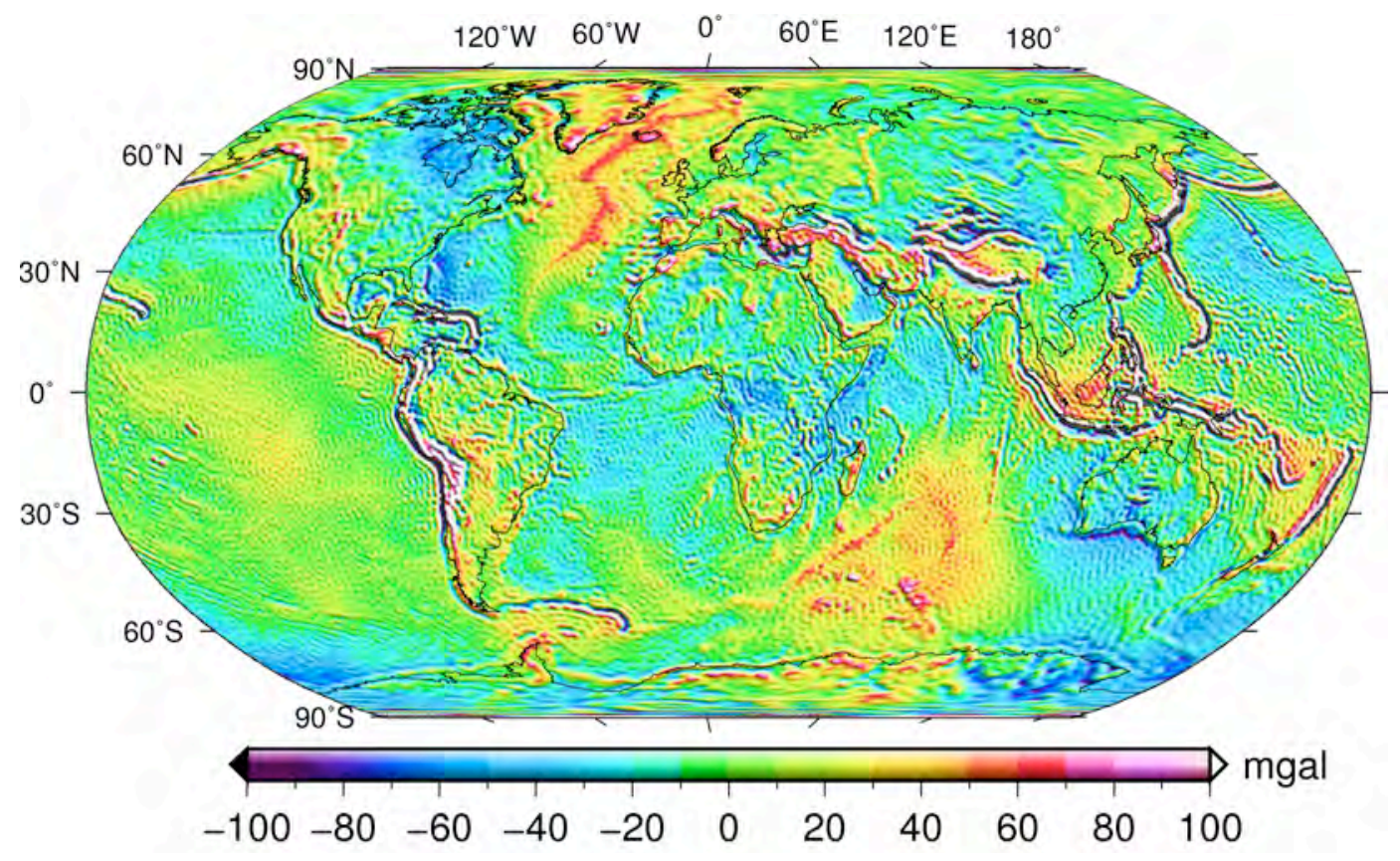

Abbildung 2: Das Schwerefeld der Erde aus GOCE, (Quelle: W.Yi, IAPG, TUM,2012) Literaturhinweise:

Journal of Geodesy: Special Issue: GOCE: The Gravity and steady-state Ocean Circulation Explorer, 85(11) 747-884, 2011.

Rummel, R.: Großer Aufwand für klein g, Physik Journal, Vol. 9, Nr. 3, pp 35-40, WileyVCH, 2010. 